\title{
Severe Ectopic Cushing's Syndrome: Feasibility of Bilateral Simultaneous Retroperitoneoscopic Adrenalectomy and Risk of Thromboembolic Events
}

\author{
Mechteld C de Jong ${ }^{1}$, Kithsiri J Senanayake², Bahram Jafar-Mohammadi ${ }^{3}$, Shahab Khan ${ }^{4}$, Radu Mihai ${ }^{5}$
}

\begin{abstract}
Aim and objective: To report our recent experience with bilateral simultaneous retroperitoneoscopic adrenalectomy for severe ectopic Cushing's syndrome (CS) and discuss the risk of severe thromboembolic events (TEs) in such patients.

Background: Patients with CS have an increased risk of arterial and venous TEs. For patients with severe ectopic CS not responding to the medical blockade, bilateral adrenalectomy is indicated to relieve symptoms.

Case description: Three patients underwent a bilateral simultaneous retroperitoneoscopic adrenalectomy for severe ectopic CS. The minimally invasive procedure was completed in all patients, with total operative times ranging from 70 to 120 minutes and negligible overall blood loss. All patients developed perioperative TEs despite being on prophylactic dosages of low molecular weight heparin (5,000 U Dalteparin). Postoperative hospital stay was prolonged (6-20 days) due to complex medical needs and difficult physical rehabilitation. During follow-up (6-20 months), mobility and functional status improved significantly in all patients. All remain on daily adrenal replacement with no biochemical signs of recurrent hypercortisolism.

Conclusion: Bilateral simultaneous retroperitoneoscopic adrenalectomy for severe ectopic CS is feasible, safe, and advantageous in selected centers with sufficient surgical expertise. The risk of developing a TE for these patients is extremely high and international guidelines should be developed.

Clinical significance: The risk of development of TE for the patient with severe ectopic CS in the presence of a disseminated malignancy is extremely high. In addition to following current guidelines on anti-TE prophylaxis, we consider that all patients should undergo a formal lower limb duplex ultrasound scan at the time of initial surgical workup, to select those patients who need to be started on early therapeutic dose anticoagulation if the presence of a TE is confirmed.

Keywords: Ectopic Cushing's syndrome, Simultaneous retroperitoneoscopic adrenalectomy, Thromboembolic events.

World Journal of Endocrine Surgery (2021): 10.5005/jp-journals-10002-1311
\end{abstract}

\section{BACKGROUND}

Cushing's syndrome (CS) is caused by chronic glucocorticoid excess which leads to considerable morbidity and mortality. Recently, there has been increased interest in defining the added risk of arterial and venous thromboembolic events (TEs) in such patients.' Specifically, in a recent study including 208 patients with CS undergoing any type of medical or surgical treatment, ${ }^{2}$ it was reported that almost one-fifth (18\%) developed a TE, with many patients developing more than one event and with equal risk of an arterial or venous TEs. The cause for this excess risk of TEs is multifactorial, with increased production of procoagulant factors like fibrinogen, factor VIII, and VWF on one side and an impaired fibrinolytic capacity, which is reflected by elevated plasma concentrations of PAI-1, TAFI, and a2-antiplasmin, on the other side. Ultimately, these changes result in a shortened APTT and a decreased fibrinolytic potential. ${ }^{3}$

Ectopic CS is a rare form of CS in which a source outside the pituitary gland produces adrenocorticotropic hormone (ACTH). A wide spectrum of primary tumors has been reported to be associated with ectopic CS, but the most common are neuroendocrine tumors (NETs) of the lung and gut. ${ }^{4}$ If the primary tumor cannot be localized or if it is diagnosed as widely disseminated/metastatic disease not amenable to curative surgical treatment, medical blockade of excess cortisol production is beneficial and various success rate has been reported with ketoconazole, aminoglutethimide, or metyrapone. ${ }^{5}$ Bilateral adrenalectomy is generally indicated when medical
1,2,4,5 Department of Endocrine Surgery, Oxford University Hospitals NHS Foundation Trust, Oxford, UK

${ }^{3}$ Department of Endocrinology, Oxford University Hospitals NHS Foundation Trust, Oxford, UK

Corresponding Author: Radu Mihai, Department of Endocrine Surgery, Oxford University Hospitals NHS Foundation Trust, Oxford, UK, Phone: +44 1865 223555, e-mail: Radu.Mihai@ouh.nhs.uk

How to cite this article:de Jong MC, SenanayakeKJ,Jafar-MohammadiB, et al. Severe Ectopic Cushing's Syndrome: Feasibility of Bilateral Simultaneous Retroperitoneoscopic Adrenalectomy and Risk of Thromboembolic Events. World J Endoc Surg 2021;13(1):16-19.

Source of support: Nil

Conflict of interest: None

therapy fails, though some consider it preferable to the long-term medical blockade, and therefore more patients should be referred for surgical treatment while they remain fit enough in order to avoid rapid deterioration in physical ability during prolonged period of uncontrolled hypercortisolism.

Currently, there are no disease-specific guidelines for perioperative prophylactic or therapeutic anticoagulation for patients with CS and the need is even more significant for the subgroup with malignant ectopic CS. 
This case series describes our recent experience with patients who underwent bilateral adrenalectomy for severe ectopic malignant CS.

\section{Case Description}

Between January 2018 and June 2020, 150 patients underwent adrenalectomy at our institution. All clinical data were collected in an institutional database. Of these patients, three patients underwent a bilateral simultaneous retroperitoneoscopic adrenalectomy for severe ectopic malignant CS.

All three patients were female, with ages ranging from 56 to 74 years. Two patients were diagnosed with a well-differentiated NET with primaries in small bowel and pancreas and both had multiple liver metastases at the time of diagnosis (Fig. 1). In the third patient, the primary lesion, and thus the source of ACTH, was not identified despite extensive cross-sectional imaging of the chest-abdomen-pelvis. Both patients with a known primary tumor location underwent combined modality treatments for NETs, including resection and chemotherapy (Fig. 2). However, for all three patients, severe ectopic CS was present and medical blockade, with metyrapone, spironolactone, and potassium-supplements, was found to be ineffective for the control of their symptoms.

The indication for surgical treatment with bilateral adrenalectomy was discussed in a regional neuroendocrine multidisciplinary meeting. At the time of the referral for surgery, patients had experienced 2-20 months of hypercortisolism and had convincing biochemical evidence of hypercortisolism (Table 1).

Bilateral adrenalectomy was recommended. Based on local expertise and previous experience with adrenal surgery,
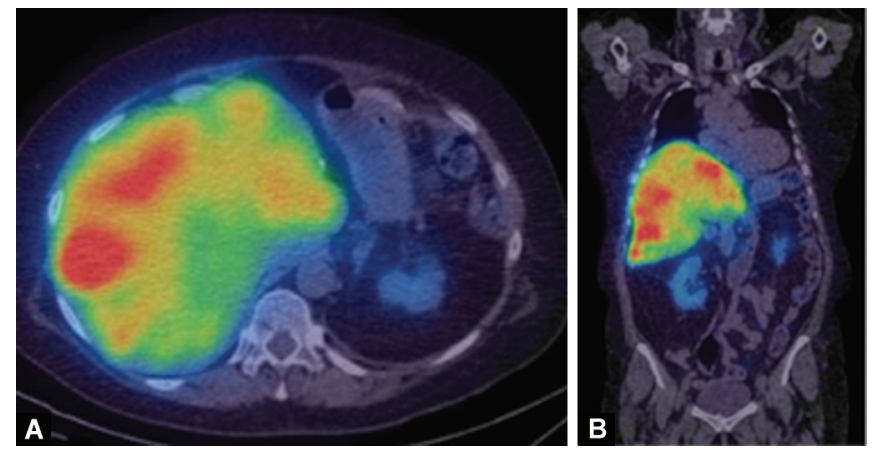

Figs $1 \mathrm{~A}$ and $\mathrm{B}$ : Fused transaxial (A) and coronal (B) images of an $\mathrm{NM}^{177} \mathrm{Lu}$ labeled somatostatin analog scan demonstrating multiple lutatate-avid bilobar liver metastases
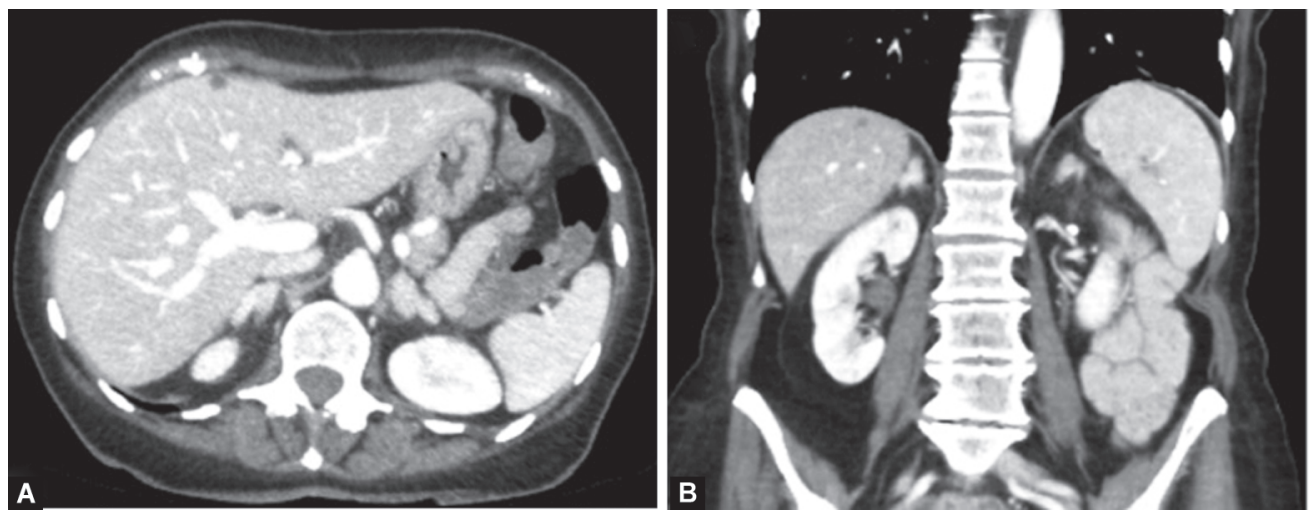

Figs 2A and B:Transaxial (A) and coronal (B) CT scan images demonstrating bilateral adrenal gland hyperplasia it was decided to perform the procedure as a simultaneous retroperitoneoscopic resection, i.e., with two full operating teams operating on either side of the patient at the same time. Patients were positioned in a knee-chest position on a modified Maquet operating table. Per side, three trocars were inserted for the simultaneous retroperitoneoscopic adrenalectomy: a $12 \mathrm{~mm}$ balloon trocar (location: the tip of the 12th rib) and two auxiliary working ports: a $10 \mathrm{~mm}$ port (location: midline between $12 \mathrm{~mm}$ trocar and spine), and a $5 \mathrm{~mm}$ port (location: the tip of the 11th rib). Insufflation was performed with $\mathrm{CO}_{2}$ at $20 \mathrm{~mm} \mathrm{Hg}$ pressures and per side, one $30^{\circ}$ camera was used. Apart from an atraumatic grasper, a $5 \mathrm{~mm}$ laparoscopic energy device and a $5 \mathrm{~mm}$ clip applier were used. The specimen was removed using a laparoscopic retrieval bag. Saline solution to wash the operative field was used on indication;

Table 1: Clinical details of patients undergoing bilateral simultaneous adrenalectomy

\begin{tabular}{|c|c|c|c|}
\hline & Patient 1 & Patient 2 & Patient 3 \\
\hline $\begin{array}{l}\text { Location of the } \\
\text { primary tumor }\end{array}$ & $\begin{array}{l}\text { Well- } \\
\text { differentiated } \\
\text { NET in the } \\
\text { pancreas }\end{array}$ & $\begin{array}{l}\text { Well- } \\
\text { differentiated } \\
\text { NET in the } \\
\text { pancreas }\end{array}$ & $\begin{array}{l}\text { Unknown at } \\
\text { the time of } \\
\text { adrenal surgery }\end{array}$ \\
\hline $\begin{array}{l}\text { The extent } \\
\text { of metastatic } \\
\text { disease }\end{array}$ & $\begin{array}{l}\text { Multiple liver } \\
\text { metastases }\end{array}$ & $\begin{array}{l}\text { Multiple liver } \\
\text { metastases }\end{array}$ & Negative \\
\hline $\begin{array}{l}\text { Preoperative* } \\
\text { plasma cortisol } \\
\text { levels, in } \mathrm{nmol} / \mathrm{L}\end{array}$ & 2170 & 2267 & 2038 \\
\hline $\begin{array}{l}\text { Preoperative* } \\
\text { 24-hour urinary } \\
\text { cortisol levels, in } \\
\text { nmol/day }\end{array}$ & 15,700 & $\mathrm{~N} / \mathrm{A}$ & 8967 \\
\hline $\begin{array}{l}\text { Relevant medical } \\
\text { therapy before } \\
\text { surgery (e.g., } \\
\text { block and } \\
\text { replace regime) }\end{array}$ & $\begin{array}{l}\text { Dalteparin } \\
5,000 \text { U OD; } \\
\text { Metyrapone } \\
750 \text { mg TD; } \\
\text { Hydrocortisone } \\
10 \text { mg BD }\end{array}$ & $\begin{array}{l}\text { Dalteparin } \\
5,000 \text { U OD; } \\
\text { Metyrapone } \\
500 \text { mg BD }\end{array}$ & $\begin{array}{l}\text { Dalteparin } \\
5,000 \mathrm{U} \text { OD; } \\
\text { Block and } \\
\text { replace the } \\
\text { regime of me- } \\
\text { tyrapone and } \\
\text { hydrocortisone }\end{array}$ \\
\hline $\begin{array}{l}\text { Postoperative } \\
\text { length of stay, in } \\
\text { days }\end{array}$ & 10 & 6 & 20 \\
\hline $\begin{array}{l}\text { Length of follow- } \\
\text { up, in months }\end{array}$ & 20 & 10 & 6 \\
\hline
\end{tabular}

${ }^{*}$ At time of initial diagnosis 

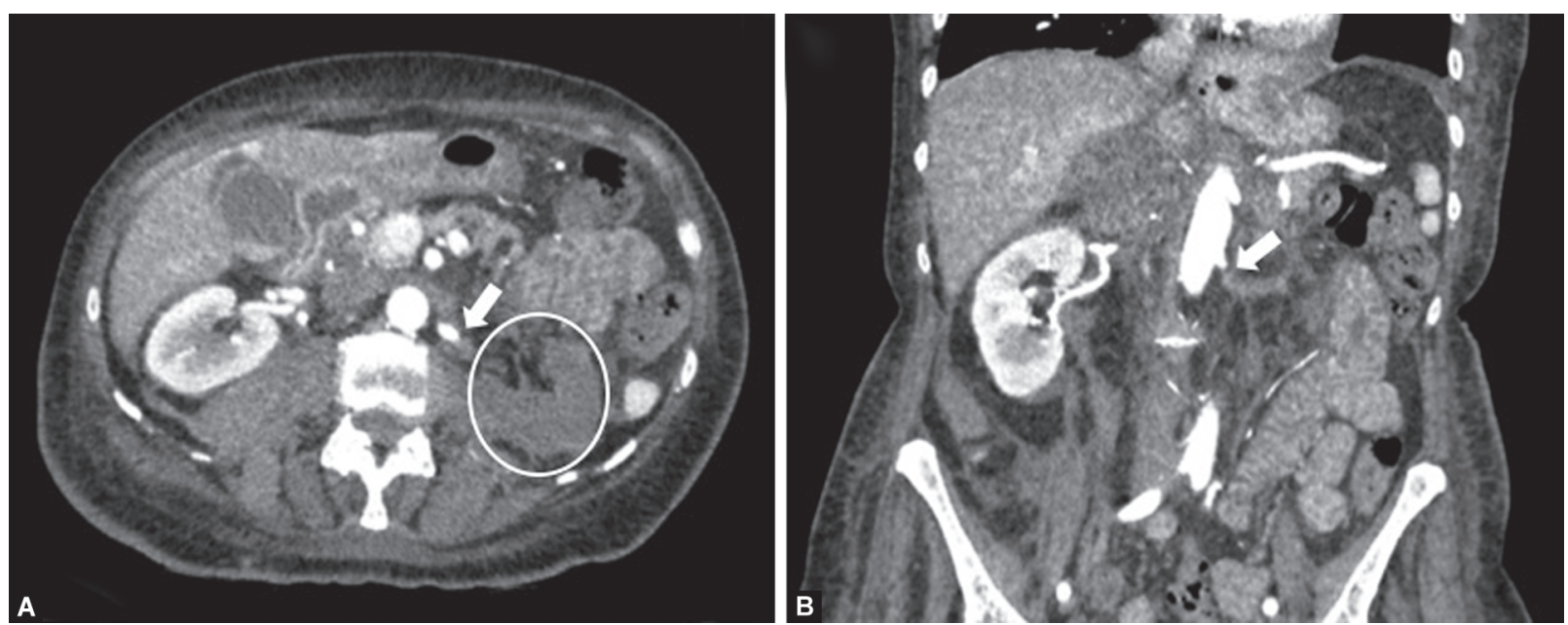

Figs $3 A$ and B: Transaxial (A) and coronal (B) CT scan images with arterial contrast, demonstrating a normal enhancing right kidney, but showing a rapid cutoff of the left renal artery (arrow) with total infarction of the kidney (circle)

no drains were placed. The closure was undertaken in layers, with a non-absorbable suture for the fascia and absorbable sutures for the skin. The procedure was completed in all patients, with total operative times ranging from 70 to 120 minutes. Overall blood loss was negligible $(<20 \mathrm{~mL})$. The operative approached followed closely the previous description of this technique by Martin Walz (reviewed by Alesina et al. ${ }^{6}$ ).

All three patients developed a TE during their postoperative recovery, despite all receiving prophylactic dosages of low molecular weight heparin before their procedure and all wore thrombo-embolus deterrent stockings (TEDs) throughout their admission in hospital as well as intermittent calf compression garments (Flowtrons) during their procedure and the first night postoperatively. Specifically, two patients developed a postoperative pulmonary embolism. One patient was considered to have had an arterial TE of the left renal artery with a subsequent total infarction of the kidney and perioperative hypertension (Fig. 3). This patient had a period of high blood pressure just before induction of anesthesia, subsequently had an apparently uneventful operation, then was suspected to have had a postoperative bleed while being investigated in the recovery room, and thus underwent a CT scan, which showed no perfusion of the left kidney. An intraoperative injury to the left renal artery was deemed very unlikely (artery seen during dissection, no clips applied, and dissection of adrenal vein was done away from the renal pedicle) and therefore acute thromboembolic occlusion of the renal artery was considered the most likely cause (unproven).

No other postoperative complication occurred and there was no mortality. Postoperative hospital stay ranged from 6 to 20 days.

During a 6-20-month postoperative follow-up, all patients improved their mobility and functional status significantly. All patients remain on daily replacement with 20 mg hydrocortisone (in divided doses) and $50 \mu \mathrm{g}$ fludrocortisone, as per institutional protocol based on national guidance, and none have biochemical evidence of hypercortisolism. A small suspicious lung nodule was identified in the one of patients as a possible source of ectopic ACTH secretion.

\section{Discussion}

We report on a series of three patients with severe ectopic CS who underwent a bilateral simultaneous retroperitoneoscopic adrenalectomy. The procedure was found to be technically safe and feasible and was deemed to be the ideal treatment in view of a shorter operating time. Overall, (synchronous) bilateral adrenalectomy is an exceedingly rare operation, as $<1 \%$ of patients undergoing adrenal surgery will need a bilateral procedure. ${ }^{7}$ In all three patients, the procedure was completed, with a total operative time ranging from 70 to 120 minutes. In literature, the mean operative time for unilateral retroperitoneoscopic adrenalectomy ranges from 40 to 106 minutes, ${ }^{8,9}$ while the operative times for simultaneous bilateral approaches seem to be somewhat longer, at a mean of 157 to 170 minutes. ${ }^{10,11}$ In general, there is no need for delayed surgery (i.e., staged surgery) when adequate surgical and anesthetic expertise exists and this operation can be achieved with minimal blood loss, in relatively short operating time and without significant morbidity. ${ }^{7}$

All three patients developed a TE following their surgery, despite prophylactic measures (i.e., prophylactic dosages of low molecular weight heparin before their procedure and TED stockings plus Flowtrons). No other complications occurred. Overall complication rates for unilateral retroperitoneoscopic adrenalectomy range from 0 to $14 \%$ and include surgical site infection, bleeding, pneumothorax, pneumonia, and temporary relaxation and/or hypesthesia of the abdominal wall. ${ }^{8,9,12}$ None of these complications were observed in our series. Moreover, the procedure of a retroperitoneoscopic adrenalectomy in itself, therefore, does not seem to be associated with an increased risk of $\mathrm{TE}$, as reported by Walz et al. in their study of 560 procedures, where none of the patients developed an iliac or femoral vein thrombosis, or pulmonary embolism. ${ }^{8}$ Furthermore, in a previous review of 23 bilateral adrenalectomy cases from our institution, ${ }^{13}$ of which 13 were for CS and of whom three underwent a retroperitoneoscopic adrenalectomy, no patient developed a TE. This could be explained by lack of awareness at that time (i.e., possible failure to diagnose subclinical disease) or possibly because all of these patients had benign disease rather than a wide-spread malignancy.

Recent studies report the risk of developing a TE for the whole group of patients with CS to approximate one-in-five, with many patients having more than one event. ${ }^{2,14}$ These numbers are corroborated in a recent review, reporting that pulmonary thrombosis has been described in up to $14 \%$ of patients with 
ectopic CS. ${ }^{4}$ To date, there has been no systematic reporting of the incidence of TEs in large cohorts of patients with ectopic CS.

Interestingly, the highest risk of developing a TE seems to be within the first 30-60 days prior to surgery, although others reported that the risk of TE may remain increased for years after surgery, even after remission from $\mathrm{CS}^{14}$ Moreover, the presence of any malignancy further predisposes patients to developing a $T E$, with some suggesting the risk of developing a venous TE to be 5-7-fold increased. ${ }^{15}$ This risk is considered to be even higher when metastatic disease is present. ${ }^{16}$ For patients with severe ectopic CS-as those described in our case series-the risk of developing a TE will thus probably be more than cumulative.

The currently available guidelines on the treatment of CS from the endocrine society do not specify the type and length of anti-TE prophylaxis, ${ }^{17}$ however, as minimal prophylaxis, the administration of low molecular weight heparin will most likely already need to be started during the surgical work-up, and to be continued until up to 60 days postoperatively. ${ }^{2}$ Similarly, the NICE guidelines advise mechanical prophylaxis (e.g., TED stockings) starting on admission and a pharmacological VTE prophylaxis for a minimum of 7 days for people undergoing abdominal surgery, continuing up to 28 days postoperatively for people who have had major cancer surgery. ${ }^{18}$ In addition to this, however, as the group of patients undergoing surgery for ectopic CS might be of an even higher risk, they might benefit from a more pro-active approach in diagnosing a possibly already present VTE-and thus an early treatment-could be considered by means of a formal lower limb DVT ultrasound scan at time of surgical workup. This advice for systematic evaluation for thrombophlebitis and pulmonary thrombosis to be performed early in severe ectopic CS is also specified by Young et al. ${ }^{4}$

\section{Conclusion}

Bilateral simultaneous retroperitoneoscopic adrenalectomy is a very rare operation, which is feasible, safe, and advantageous in selected centers with sufficient surgical expertise. In the absence of international guidelines for perioperative TE prophylaxis for this high-risk group with disseminated malignancy and severe ectopic CS, our advice would be for all patients to have a formal comprehensive duplex ultrasound scan from thigh to ankle at time of their surgical workup. This can help select those patients who need to be started on therapeutic dose anticoagulation if TE is diagnosed.

\section{Clinical Significance}

The risk of development of a TE for patients with severe ectopic CS in the presence of a disseminated malignancy is extremely high and imposes management uncertainties especially in the absence of international guidelines. The current report describes the outcomes of a series of three patients with this disease who underwent bilateral simultaneous retroperitoneoscopic adrenalectomy. All included patients developed a $\mathrm{TE}$, although receiving prophylactic dosages of low molecular weight heparin, whereas no other postoperative complications occurred. The performance of a bilateral simultaneous retroperitoneoscopic adrenalectomy is therefore deemed feasible and safe. For patients undergoing this procedure, we would recommend performance of a formal lower limb duplex ultrasound scan at time of surgical workup, to select those patients be started on therapeutic doses anticoagulation if a TE is diagnosed.

\section{References}

1. Wagner J, Langlois F, Lim DST, et al. Hypercoagulability and risk of venous thromboembolic events in endogenous Cushing's syndrome: a systematic meta-analysis. Front Endocrinol (Lausanne) 2018;9:805. DOI: 10.3389/fendo.2018.00805.

2. Suarez MG, Stack M, Hinojosa-Amaya JM, et al. Hypercoagulability in cushing syndrome, prevalence of thrombotic events: a large, single-center, retrospective study. J Endocr Soc 2020;4(2):bvz033. DOI: 10.1210/jendso/bvz033.

3. van der Pas R, Leebeek FW, Hofland LJ, et al. Hypercoagulability in Cushing's syndrome: prevalence, pathogenesis and treatment. Clin Endocrinol (Oxf) 2013;78(4):481-488. DOI: 10.1111/cen.12094.

4. Young J, Haissaguerre M, Viera-Pinto $O$, et al. Management of endocrine disease: cushing's syndrome due to ectopic ACTH secretion: an expert operational opinion. Eur J Endocrinol 2020;182(4):R29-R58. DOI: 10.1530/EJE-19-0877.

5. Feelders RA, Newell-Price J, Pivonello R, et al. Advances in the medical treatment of Cushing's syndrome. Lancet Diabetes Endocrinol 2019;7(4):300-312. DOI: 10.1016/S2213-8587(18)30155-4.

6. Alesina PF. Retroperitoneal adrenalectomy-learning curve, practical tips and tricks, what limits its wider uptake. Gland Surg 2019;8(Suppl 1):S36-S40. DOI: $10.21037 / \mathrm{gs} .2019 .03 .11$.

7. Mihai R. Bilateral adrenalectomy—simultaneous or delayed? Laparosc Surg 2019. 3. DOI: $10.21037 / \mathrm{ls}$.2019.06.03

8. Walz MK, Alesina PF, Wenger FA, et al. Posterior retroperitoneoscopic adrenalectomy--results of 560 procedures in 520 patients. Surgery 2006;140(6):943-948. DOI: 10.1016/j.surg.2006.07.039; discussion 8-50.

9. Costa Almeida CE, Caroço T, Silva MA, et al. Posterior retroperitoneoscopic adrenalectomy-case series. Int J Surg Case Rep 2018;51:174-177. DOI: 10.1016/j.ijscr.2018.08.044.

10. Raffaelli M, Brunaud L, De Crea C, et al. Synchronous bilateral adrenalectomy for Cushing's syndrome: laparoscopic versus posterior retroperitoneoscopic versus robotic approach. World J Surg 2014;38(3):709-715. DOI: 10.1007/s00268-013-2326-9.

11. Miccoli P, Materazzi G, Brauckhoff M, et al. No outcome differences between a laparoscopic and retroperitoneoscopic approach in synchronous bilateral adrenal surgery. World J Surg 2011;35(12):26982702. DOI: 10.1007/s00268-011-1294-1.

12. Lee $C R$, Walz MK, Park S, et al. A comparative study of the transperitoneal and posterior retroperitoneal approaches for laparoscopic adrenalectomy for adrenal tumors. Ann Surg Oncol 2012;19(8):2629-2634. DOI: 10.1245/s10434-012-2352-0.

13. Maccora D, Walls GV, Sadler GP, et al. Bilateral adrenalectomy: a review of 10 years' experience. Ann R Coll Surg Engl 2017;99(2):119-122. DOI: 10.1308/rcsann.2016.0266.

14. Dekkers OM, Horváth-Puhó E, Jørgensen JOL, et al. Multisystem morbidity and mortality in Cushing's syndrome: a cohort study. J Clin Endocrinol Metab 2013;98(6):2277-2284. DOI: 10.1210/jc.2012-3582.

15. Abdol Razak NB, Jones G, Bhandari M, et al. Cancer-associated thrombosis: an overview of mechanisms, risk factors, and treatment. Cancers (Basel) 2018;10(10):380. DOI: 10.3390/cancers10100380.

16. Fernandes CJ, Morinaga LTK, Alves JL, et al. Cancer-associated thrombosis: thewhen, how and why. Eur Respir Rev 2019;28(151):180119. DOI: 10.1183/16000617.0119-2018.

17. Nieman LK, Biller BM, Findling JW, et al. Treatment of Cushing's syndrome: an endocrine society clinical practice guideline. J Clin Endocrinol Metab 2015;100(8):2807-2831. DOI: 10.1210/jc.2015-1818.

18. NICE Guidelines - Venous thromboembolism in over 16s: reducing the risk of hospital-acquired deep vein thrombosis or pulmonary embolism. London: National Institute for Health and Care Excellence (UK). National Institute for Health and Care Excellence: Clinical Guidelines. 2019 Aug 13 ed 2019. 